\title{
Corrections
}

\section{Correction: Sasaki et al., Functional Differentiation of a Population of Electrically Coupled Heterogeneous Elements in a Microcircuit}

In the article "Functional Differentiation of a Population of Electrically Coupled Heterogeneous Elements in a Microcircuit" by Kosai Sasaki, Elizabeth C. Cropper, Klaudiusz R. Weiss, and Jian Jing, which appeared on pages 93-105 of the January 2, 2013 issue, the authors regret misspelling the first author's first name. The corrected author line is: Kosei Sasaki, Elizabeth C. Cropper, Klaudiusz R. Weiss, and Jian Jing, which has been corrected on the online PDF version.

DOI: 10.1523/JNEUROSCI.0062-13.2013

\section{Correction: Chen et al., Alert Response to Motion Onset in the Retina}

In the article "Alert Response to Motion Onset in the Retina" by Eric Y. Chen, Olivier Marre, Clark Fisher, Greg Schwartz, Joshua Levy, Rava Azeredo da Silviera, and Michael J. Berry II, which appeared on pages 120-132 of the January 2, 2013 issue, the authors regret misspelling the sixth author's last name. The corrected author line is: Eric Y. Chen, Olivier Marre, Clark Fisher, Greg Schwartz, Joshua Levy, Rava Azeredo da Silveira, and Michael J. Berry II which has been corrected on the online PDF version.

DOI: $10.1523 / J N E U R O S C I .0160-13.2013$ 